\title{
Modeling, Vibration Analysis and Fabrication of Micropumps Based on Piezoelectric Transducers
}

\author{
Yanfang Guan ${ }^{\dagger}$, Xiangxin Meng, Yansheng Liu, Mingyang Bai and Fengqian Xu \\ School of Electromechanical Engineering, Henan University of Technology, Zhengzhou 450001, China. \\ $\dagger$ Corresponding author
}

\begin{abstract}
(Received 28 October 2019; accepted 23 January 2020)
The parametric and vibrational characteristics of PZTs (Piezoelectric Transducers) with different diameters before and after coupling are discussed by finite element analysis. It is shown that the vibration stability of the piezoelectric transducer decreases with increasing driving frequency. The PZT's variation of maximum displacement with frequency shows the same trend for different driving conditions according to vibration measurement under conditions of both free and forced vibration (before and after sealing with the pump body). The maximum displacement under forced vibration is less than that under free vibration. The maximum displacement is inversely proportional to the diameter of the transducer and directly proportional to the driving voltage under both free and forced vibration. Micropumps with diffuser/nozzle microvalves are designed and fabricated with different external diameters of the PZTs. Finally, the flow rate and pressure of the micropumps are measured, which are consistent with the vibrational results. Moreover, the maximum displacement is larger under a square-wave driving signal, followed by a sine-wave signal, and then a triangle-wave signal. For a PZT with an external diameter of $12 \mathrm{~mm}$, the maximum flow rate and pressure value are $150 \mu \mathrm{l} / \mathrm{min}$ and $346 \mathrm{~Pa}$, respectively, under sine-wave driving at 100 Vpp driving voltage.
\end{abstract}

\section{INTRODUCTION}

Piezoelectric transducers are the devices that use the piezoelectric properties of some crystals or other materials to convert one type of energy to another one. Moreover, piezoelectric transducers are often used as driving devices for actuating microfluidic systems, since flow inside a microfluidic device could be affected by vibration. ${ }^{1-3}$ Therefore, it is important to study the behavior of piezoelectric transducers, especially when they are coupled with microfluidic devices. ${ }^{4-6}$ Specifically, in practical applications, it is very important to understand the influence of transducer vibration on microfluidics performance. ${ }^{7-10}$ For example, Liu ${ }^{11}$ studied the coupled vibration of a sandwich piezoelectric transducer using an approximate analytical method. The results showed that the resonant frequencies obtained from the coupled resonant frequency equations are in good agreement with those from numerical methods, and super harmonic resonant frequencies can also be obtained. Zhu et al. ${ }^{12}$ simulated and analyzed the behavior of a piezoelectric flexible plate using MATLAB and ANSYS software. The vibration control experiments were performed based on an APDL (the design language of ANSYS) program. The designed controller had good vibration suppression performance according to the analysis and experimental results. Catarino et al. ${ }^{13}$ proposed a microfluidic mixing device with two different piezoelectric materials. The mixing-time reduction for both materials, above $90 \%$ for PZT and above $80 \%$ for $\beta$-PVDF, were tested according to numerical simulations and experimental studies. Huang et al. ${ }^{14}$ studied a piezoelectric harvester's resonant frequencies and voltage output equation at various excitation frequencies according to numerically calculated results as well as experiments, which showed a high level of voltage output.

Piezoelectric micropumps are popular in microfluidic systems. They have been used in medicine, agriculture, and aviation, among other applications. ${ }^{15-21}$ Therefore, it is extremely important to study how to improve the performance of micropumps, and researchers have made a lot of efforts in studying the performance of a common piezoelectric material: Lead Zirconate Titanate (PZT). Aggarwal et al. ${ }^{22}$ fabricated and studied two different sizes of piezoelectrically actuated micromachined silicon valveless micropumps with a vertical diffuser/nozzle microchannel, and the flow rate and the back pressure were evaluated. Cheng et al. ${ }^{23}$ fabricated an impedance micropump with nickel electroforming components, a stainless steel vibration plate, and a piezoelectric actuator. The flow rate of $0.24 \mathrm{ml} / \mathrm{min}$ and back pressure of $2.35 \mathrm{kPa}$ were demonstrated with a $200 \mathrm{Vpp}$ driving voltage. He et al. ${ }^{24}$ presented a new type of valveless piezoelectric micropump with synthetic jet and Coanda effect to achieve larger and bidirectional flow rate. An optimal frequency of $50 \mathrm{~Hz}$ and a Reynolds number of 1000 was identified for a maximum flow rate of $6.8 \mathrm{ml} / \mathrm{min}$. Wang et al. ${ }^{25}$ presented a Finite Element Analysis (FEA) micropump model actuated by a piezoelectric actuator. The maximum displacement of the PZT ceramic disk was found to vary along with the diameter ratio, membrane thickness and the diameter of the chamber. Kang and Auner $^{26}$ designed a piezoelectrically actuated check valve diaphragm micropump and studied the average displacement of the diaphragm. The relationship between the stroke volume and backpressure were simulated with an FEA tool. Singh et al. ${ }^{27}$ fabricated and tested a piezoelectrically actuated polydimethylsiloxane (PDMS) planar valveless micropump. The predictions of the analytical model and numerical simulations in terms of flow rate versus frequency, voltage and pressureflow matched with experimental data (within 20\%). Zhang ${ }^{28}$ presented a self-sensing piezoelectric pump with a bimorph transducer. The output flow and pressure could be achieved through a single piezoelectric element, and the simultaneous function could be achieved by the other PZT disk.

We can see that previous research has concentrated on FEA modeling of piezoelectric transducers and new structures of 


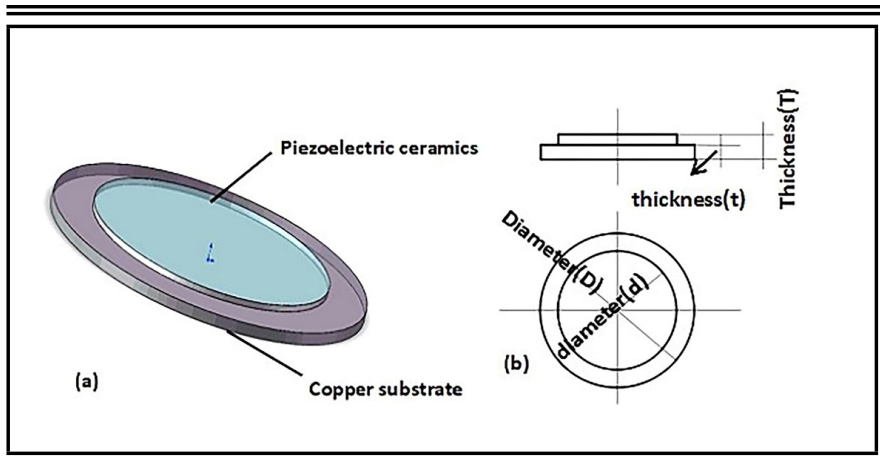

Figure 1. Structure of the piezoelectric transducer.

piezoelectric micropumps, with leads no studies on the vibrational performance of the piezoelectric transducer, especially the comparison between before and after the transducers being coupled with micropumps. To the best of our knowledge, the factors that influence the vibration of PZT before and after coupling to a micropump as well as the influence on the pumping performance have not been studied. Note that, as the main power supply device of the micropump, the vibration characteristics of PZT has an important influence on the output performance of micropump, such as the flow rate, pressure and efficiency. Through the vibrational analysis of the PZT before coupling, the optimal working conditions of PZT are obtained for further guiding the influence of PZT after coupling on the working effect of the micropump. Meanwhile, the optimal parameters combination for the micropump performance obtained during the numerical analysis realize the optimization output of the micropump performance and reduce the economic loss and simplify the operation. Therefore, in this paper, we study the vibrational performance of the piezoelectric transducer and discuss thoroughly the working principles and the influence on the performance of the micropump. The optimal PZT structures, the flow rate and pressure of the micropump are achieved according to the vibrational analysis and experiments.

\section{DESIGN AND FABRICATION}

\subsection{Structural Analysis of The Piezoelectric Transducer}

Ideally, the material of a piezoelectric transducer needs to have high reliability, a wide frequency response range, a linear response to the applied voltage, and reasonably low cost. ${ }^{29-31}$ PZT-5H piezoelectric ceramic plates with different diameters were adopted in this study. ${ }^{32,33}$ The three-dimensional structure of the piezoelectric transducer is shown in Fig. 1(a). It consists of three parts: a PZT piezoelectric ceramic, a connecting layer, and a copper substrate. Depending on the pump chamber dimensions, three different piezoelectric actuators were used, with external diameters (i.e., the diameter of the copper substrate) of 12,15 , and $20 \mathrm{~mm}$. The parameters of each of these transducers are shown in Fig. 1(b) and Table 1.

\subsection{Structural Design and Fabrication of The Micropump}

The working principle of the micropump with a diffuser/nozzle microchannel is schematically represented in Fig. 2. First of all, there is no force on the PZT without voltage applied as shown in Fig. 2(a). In the "supply mode," the piezoelectric transducer vibrates vertically upwards, increasing the

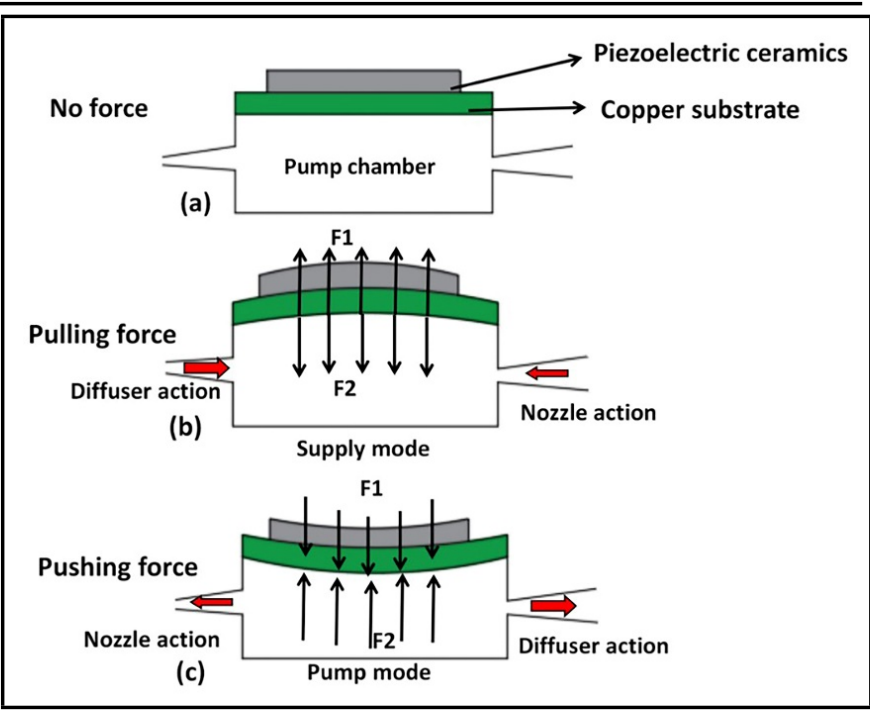

Figure 2. Working principle of the valveless micropump and the force diagram of the piezoelectric transducer. (a) No force. (b) Pushing force \& supply mode. (c) Pulling force \& pump mode.

chamber volume and thereby reducing the chamber pressure. The asymmetric arrangement of the inlet/outlet microchannel of the pump chamber enables a pressure difference to form between the pump chamber and the inlet/outlet. Then, the working fluid is sucked into the pump chamber from both the inlet (diffuser direction) and the outlet (nozzle direction), as shown in Fig. 2(b). However, the rate of the flow in the diffuser direction is larger than that of the nozzle direction as shown with red arrows of different sizes in Fig. 2(b). For the "pump mode," the reverse phenomenon occurs, as shown in Fig. 2(c). The effectiveness of the flow rectification of the micropump can be produced based on the net flow of the fluid from the inlet to the outlet if a reasonable design is adopted. ${ }^{34-37}$ The force F1 of the PZT from the transformation of electromechanical energy by the piezoelectric transducer is transmitted to the fluid in the pump chamber. At the same time, the force F2 from the fluid has a counter effect in the opposite direction. As far as the present investigation is concerned, the pump body and the piezoelectric actuator are the most important components.

The design of the piezoelectric micropump with a diffuser/nozzle microchannel is displayed in Fig. 3. As shown in Fig. 3(a), the five-layered structure of the micropump consists of, from top to bottom, an upper jig, an upper PDMS layer with inlet and outlet pipes, a glass wafer, a silicon wafer, a piezoelectric actuator, a lower PDMS layer, and a lower jig. A plan view of the nozzle/diffuser microchannel with a $7^{\circ}$ diffuser angle is shown in Fig. 3(b). A minimum section width of $0.04 \mathrm{~mm}$ and a length of $1.093 \mathrm{~mm}$ were obtained according to the advanced silicon deep reactive ion etching (DRIE) technique on $450 \mu$ m-thick silicon wafers ${ }^{34,38-40}$ in Fig. 4. The depths of the microchannel and inlet/outlet holes were $0.1 \mathrm{~mm}$, and the depth of the center pump chamber was $0.45 \mathrm{~mm}$.

The dimensions of the pump chamber and the silicon layer are shown in Fig. 3(b) together with a scanning electron microscope (SEM) image of part of the pump containing a microchannel in Fig. 3(c). A high-temperature bonding technique was used to produce an irreversible seal between the Pyrex 7740 glass wafer and the silicon wafer. The piezoelectric transducer was fixed to the lower side of the silicon wafer with $3 \mathrm{M}$ glue for total sealing. Finally, all five layers were sealed irreversibly together by ultraviolet light irradiation through the lower PDMS layer as shown in Fig. 3(d). In order to prevent 
Table 1. Parameters of the piezoelectric transducers.

\begin{tabular}{|c|c|c|c|c|c|c|}
\hline Parameter & \multicolumn{6}{|l|}{ Value } \\
\hline Piezoelectric plate diameter & \multicolumn{6}{|l|}{$11.3 \mathrm{~mm}$} \\
\hline Piezoelectric plate thickness & \multicolumn{6}{|l|}{$0.20 \mathrm{~mm}$} \\
\hline Piezoelectric coefficient matrix $\left(\mathrm{C} / \mathrm{m}^{2}\right)$ & {$\left[\begin{array}{cr}0 & 0 \\
0 & 0 \\
-6.5 & -6\end{array}\right.$} & & $\begin{array}{l}0 \\
0 \\
3.3\end{array}$ & $\begin{array}{c}0 \\
17 \\
0\end{array}$ & $\begin{array}{l}17 \\
0 \\
0\end{array}$ & $\left.\begin{array}{l}0 \\
0 \\
0\end{array}\right]^{T}$ \\
\hline Permittivity $\epsilon(\mathrm{F} / \mathrm{m})$ & {$\left[\begin{array}{cc}1.7 & 0 \\
0 & 1.7 \\
0 & 0\end{array}\right.$} & $\begin{array}{c}0 \\
0 \\
1.58\end{array}$ & \multicolumn{4}{|c|}{$\times 10^{11}$} \\
\hline Young's modulus $(\mathrm{Pa})$ & {$\left[\begin{array}{cc}1.7 & 0 \\
0 & 1.7 \\
0 & 0\end{array}\right.$} & $\begin{array}{c}0 \\
0 \\
1.58\end{array}$ & \multicolumn{4}{|c|}{$\times 10^{11}$} \\
\hline Poisson's ratio & \multicolumn{6}{|c|}{$\lambda_{12}=\lambda_{13}=\lambda_{23}=0.3$} \\
\hline Shear modulus $(\mathrm{Pa})$ & {$\left[\begin{array}{cc}0 & 2.33 \\
0 & 0 \\
0 & 0\end{array}\right.$} & $\left.\begin{array}{c}2.3 \\
2.3 \\
0\end{array}\right]$ & \multicolumn{4}{|c|}{$\times 10^{10}$} \\
\hline Density $\left(\mathrm{kg} / \mathrm{m}^{3}\right)$ & \multicolumn{6}{|l|}{$\frac{L}{7500}$} \\
\hline Copper substrate diameter & \multicolumn{6}{|c|}{$12,15,20 \mathrm{~mm}$} \\
\hline Copper substrate thickness & \multicolumn{6}{|c|}{$012,0.37,0.47 \mathrm{~mm}$} \\
\hline Copper Young's modulus $(\mathrm{Pa})$ & \multicolumn{6}{|c|}{$0.9 \times 10^{11}$} \\
\hline Copper Poisson's ratio & \multicolumn{6}{|l|}{0.32} \\
\hline Copper density $\left(\mathrm{kg} / \mathrm{m}^{3}\right)$ & \multicolumn{6}{|c|}{8500} \\
\hline Resonant frequency & \multicolumn{6}{|c|}{$5.3 \pm 0.5 \mathrm{kHz}, 10.0 \pm 0.5 \mathrm{kHz}, 7.2 \pm 0.5 \mathrm{kHz}$} \\
\hline Impedance & \multicolumn{6}{|c|}{$500,300,300 \Omega$} \\
\hline Capacitance & \multicolumn{6}{|c|}{$15 \mathrm{nF} \pm 30 \%, 9 \mathrm{nF} \pm 30 \%, 12 \mathrm{nF} \pm 30 \%$} \\
\hline
\end{tabular}

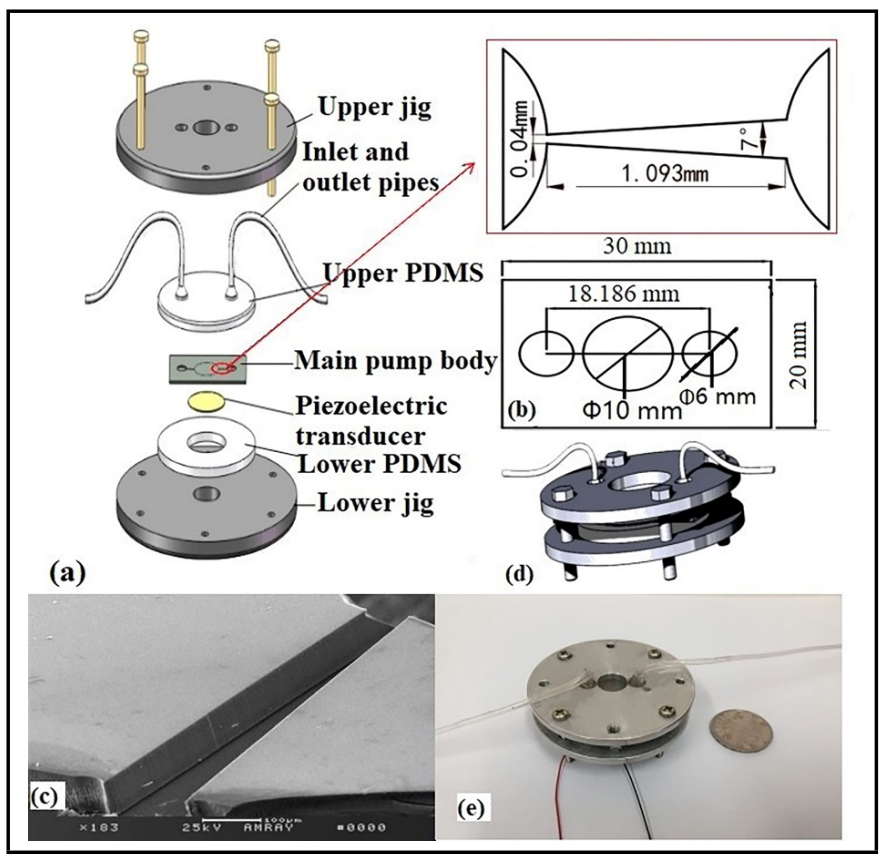

Figure 3. Structure diagram of the piezoelectric micropump. (a) Exploded view of the whole micropump. (b) Two-dimensional structure of the microchannel and pump body. (c) Plan view of a microchannel and SEM image of a microchannel. (d) Structural parameter of the pump body. (e) The photograph of complete pump assembly.

leakage, the upper and lower jigs were added to fix the micropump body. A photograph of the complete pump assembly is shown in Fig. 3(e).

\section{SIMULATION AND VIBRATIONAL ANALYSIS OF THE MICROPUMP}

Numerical modeling has been often adopted for solving three-dimensional fluid problems using CFD-ACE+ software based on the finite volume analysis, which include solid, fluid, and mechanical/electric field coupling calculations. However, the core of numerical analysis is solving partial differential

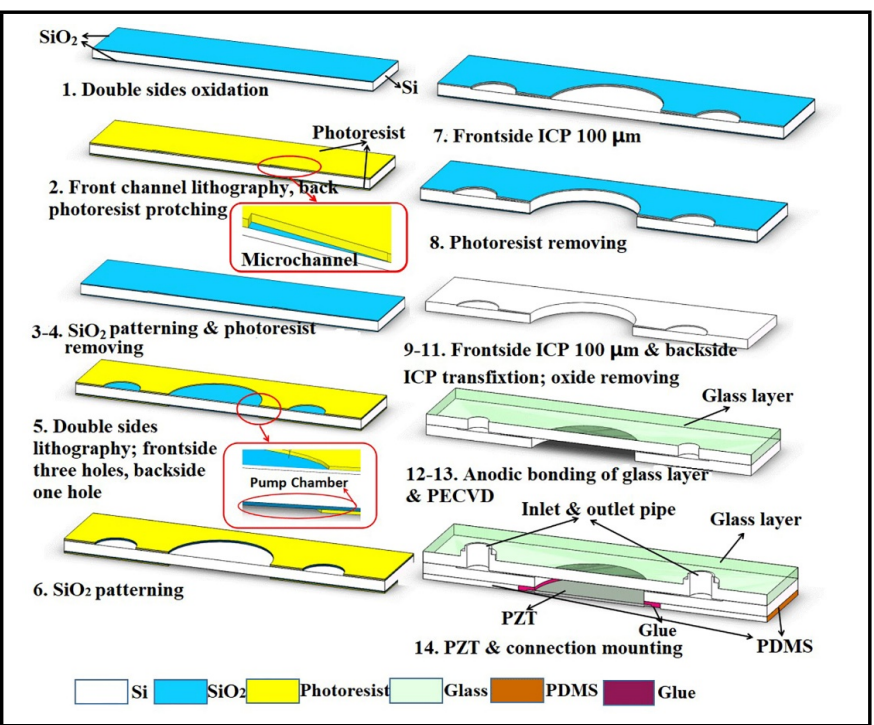

Figure 4. The fabrication process of the microchannels, pump chambers and inlet/outlet with DRIE technology (step 1-11). The anodic bonding between glass layer and silicon wafer (step 12-13). The PZT and inlet/outlet pipe connection mounting, irreversible bonding between pump body and PDMS layers (step 14).

equations. The forces on the piezoelectric transducer during micropump operation are shown in Fig. 2. The piezoelectric transducer has transverse contraction under the sine-wave alternating voltage. The force F1 from the transformation of electromechanical energy by the piezoelectric transducer will be transmitted to the fluid in the pump chamber. At the same time, the force F2 from the fluid has a counter effect in the opposite direction. The physical parameters of the piezoelectric transducer used in calculation are listed in Table 1.

The flow into the micropump is considered as incompressible and laminar. Therefore, for numerical modeling, the electromechanical-coupled equation, piezoelectric transducer control equation, Navier-Stokes equation, and continuity equation are as follows: ${ }^{41}$

$$
\sigma=C \varepsilon-e E
$$




$$
\begin{gathered}
D \nabla^{4} W+\rho h \frac{\delta^{2} W}{\delta t^{2}}=f-F_{1} ; \\
\frac{\rho D \vec{\nu}}{D t}=\rho \vec{g}+\mu \nabla^{2} \vec{\nu}-F 2 ; \\
\frac{\delta \rho}{\delta t}+(\vec{\nu} \cdot \nabla) \rho=0 .
\end{gathered}
$$

The boundary conditions of the piezoelectric ceramic sheet at a round clamp are as follows:

$$
\begin{gathered}
\frac{\delta^{2} W}{\delta x^{2}}=\frac{\delta^{2} W}{\delta y^{2}}=0 ; \\
\frac{\delta W}{\delta t}=0 ;
\end{gathered}
$$

where $\varepsilon$ is the mechanical stretch tensor, $\sigma$ is the mechanical pressure tensor, $E$ is the electric vector, $e$ is the piezoelectric continuum tensor, $C$ is the elastic stiffness constant tensor, $\rho$ is the fluid density, $\rho=1 \times 10^{3} \mathrm{~kg} / \mathrm{m}^{3}$, $\mathrm{v}$ is the velocity vector, $\mu$ is kinetic viscosity, and $\mu=1.4 \times 10^{(-3)} \mathrm{Pa} \cdot \mathrm{s}$.

The boundary conditions of the piezoelectric transducer are the fixed periphery, and no slip occurs at the interface between the fluid and solid and the free surface for the inlet/outlet. Hence, the boundary control equations are the same as Eqs. (5),(6). By solving Eqs. (1),(2),(3),(4),(5),(6) the PZTMembrane-Fluid coupled analysis can be obtained. In addition, considering that the initial stress $(\sigma)$ has a great influence on the electromechanical coupling constant of PZT, ${ }^{42}$ and when the value of initial stress exceeds $150 \mathrm{MPa}$ according to literature, ${ }^{42}$ the coupling constant will change abruptly due to the non-dimensional wavenumber, so the value of initial stress is set to $150 \mathrm{MPa}$ in the simulation.

The displacement curves of the piezoelectric vibrator before (free-vibration) and after (forced-vibration) coupling with the micropump in the $\pm \mathrm{Z}$ directions under three driving frequencies $(100 \mathrm{~Hz}, 1000 \mathrm{~Hz}$ and $5000 \mathrm{~Hz}$, respectively) are shown in Fig. 5. The $12 \mathrm{~mm}$ of external diameter and the related parameters are adopted during finite element analysis. Note that, free-vibration means the vibration of the piezoelectric transducer occurs uncoupled or unsealed with the micropump (marked as PZT-100 Hz/1000Hz/5000Hz in Fig. 5), and forced-vibration means the vibration of the piezoelectric transducer occurs coupled and sealed with the micropump (marked as pump- $100 \mathrm{~Hz} / 1000 \mathrm{~Hz} / 5000 \mathrm{~Hz}$ in Fig. 5); and the latter is the real working situation. It can be seen that the displacement of the piezoelectric vibrator shows the same sinusoidal trend before and after coupling with the pump with $100 \mathrm{~Hz}$, $1000 \mathrm{~Hz}$ and $5000 \mathrm{~Hz}$ of sine wave driving frequencies under the same time step (100 time steps as shown in Fig. 5 with $\mathrm{X}$ label). Comparing the $\pm \mathrm{Z}$ directions displacements (both free- and forced-vibration modes) between $100 \mathrm{~Hz}, 1000 \mathrm{~Hz}$, and $5000 \mathrm{~Hz}$ of driving frequencies, the coincidence with sinusoidal variation is very good under the lowest driving frequency $(100 \mathrm{~Hz})$ because F2 has little effect on the vibration of the piezoelectric transducer in Fig. 2. Additionally, the displacements in the $+\mathrm{Z}$ and $-\mathrm{Z}$ directions are the same. However, when the frequency increases and reaches a certain value, like $5000 \mathrm{kHz}$ (the eigenfrequency of PZT-5H is $5.3 \pm 0.5 \mathrm{kHz}$ as shown in Table. 1), the displacements of $\pm \mathrm{Z}$ directions distort seriously. Because the force F2 that liquid exerts on the piezoelectric vibrator acts at a disadvantage with respect to the driving force of the piezoelectric vibrator F1 in Fig. 2. Moreover, the liquid force F2 plays a significant role when the frequencies become larger, which increases the deformation of

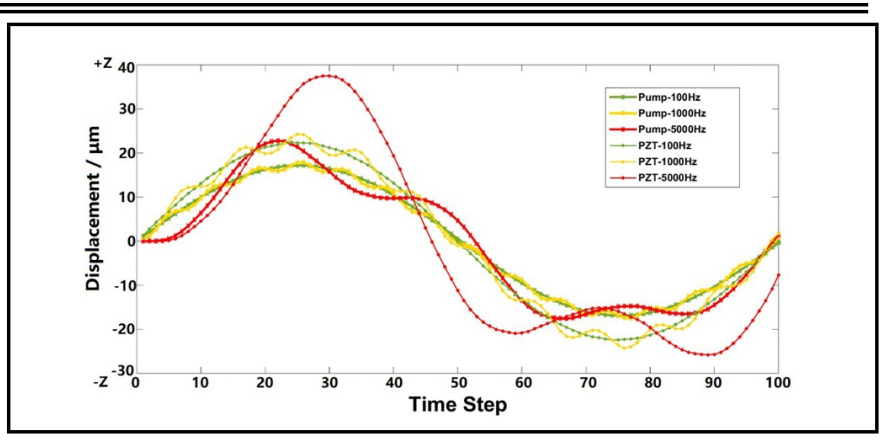

Figure 5. The displacements of the PZT before and after coupling with the pump body.

the piezoelectric transducer and the volume flow of the fluid inside the pump (It doesn't mean the net flow of the micropump in Fig. 1) also become larger. The average deformation value of the piezoelectric transducer after coupling is smaller than that before coupling. This is because the lower surface of the piezoelectric vibrator is a free vibration surface before coupling, that is, zero loads. The force F2 that liquid exerts on the piezoelectric surface blocks the piezoelectric transducer vibration after coupling (Fig. 5), so the induced deformation value decreases. For example, comparing the displacements of PZT$5000 \mathrm{~Hz}$ with Pump-5000Hz, the high frequency $(5000 \mathrm{~Hz})$ is closer to the mechanical resonance frequency of the piezoelectric actuator $(5.3 \pm 0.5 \mathrm{kHz})$, the displacement of the PZT after fixed with the pump (Pump-5000Hz) greatly reduced (close to that of the low frequency, such as $100 \mathrm{~Hz}$ ) from that of the free-vibration mode (PZT-5000Hz).

The contour maps of the pressure on the central cross section chamber under driving frequencies of $100 \mathrm{~Hz}, 1000 \mathrm{~Hz}$, and $5000 \mathrm{~Hz}$ are shown in Fig. 6 (For convenience, the quarter cycle maps of the whole period is selected because the maximum displacement occurred at that point with sine wave driving). The pressures (from 0 to $1.453 \mathrm{~N} / \mathrm{m}^{2}$ ) are positive because the maximum displacement occurred in the positive direction of quarter cycle under the sine-wave signal driving (Fig. 5) with the $100 \mathrm{~Hz}$ driving frequency (Fig. 6(a)), and there are small amounts of negative pressures (from -14.67 to $0 \mathrm{~N} / \mathrm{m}^{2}$ ) under the $1000 \mathrm{~Hz}$ driving frequency (Fig. 6(b)) because of the uneven vibration is appeared with high driving frequency (Fig. 5). However, under the $5000 \mathrm{~Hz}$ driving frequency (Fig. 6(c)), a large area of negative pressure (from -1335 to $0 \mathrm{~N} / \mathrm{m}^{2}$ ) dominates in the center of the chamber, which results in an insufficient fluid-filled micro-pump, and the pressure of the chamber accelerates with messy, inconsistent directions. It further proves that the higher frequency is not suitable as the working frequency for the micropump, i.e. near the mechanical resonance frequency. The velocity contours of the + Z-direction (or $+\mathrm{W}$-directions in Fig. $6 \mathrm{~d}$ ) should be upward (from 0 to $+0.03774 \mathrm{~m} / \mathrm{s}$ ), but some contours are downward (from $-0.01892 \mathrm{~m} / \mathrm{s}$ to $0 \mathrm{~m} / \mathrm{s}$ ) during the filling process in Fig. 6(d). This indicates the decreased efficiency of the micropump. The velocity contours of the X-direction (parallel with the fluids flow) below $5000 \mathrm{~Hz}$ frequencies are shown in Fig. 6(e). The $+Z$ direction deformation maps of the PZT with quarter cycle under $100 \mathrm{~Hz}$ and $5000 \mathrm{~Hz}$ driving frequencies are shown in Fig. 6(f) and Fig. 6(g), respectively. The deformation of the piezoelectric transducer appears hemispherical, the displacement in the central point is the largest, and the displacement in the perimeter is the smallest; this result is consistent with the boundary conditions. The deformation of every point is uniformly distributed in the plane at $100 \mathrm{~Hz}$. However, 


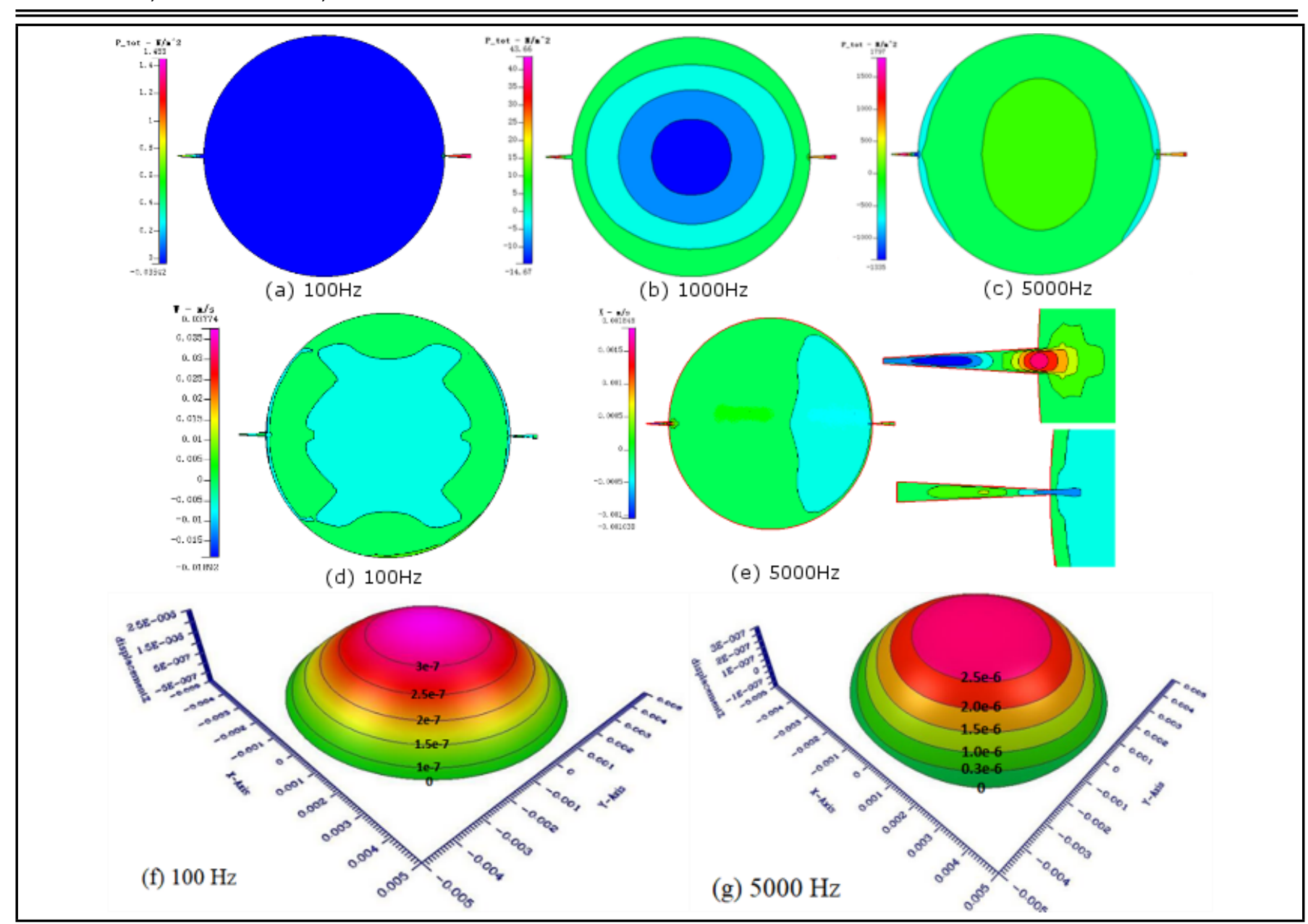

Figure 6. The contour map of the pressure on the central cross section of the chamber in the Z-direction during a quarter cycle under different driving frequency. (a) Contour map of the pressure on the central plane with driving frequency of $100 \mathrm{~Hz}$. (b) Contour map of the pressure on the central plane with driving frequency $1000 \mathrm{~Hz}$. (c) Contour map of the pressure on the central plane with driving frequency $5000 \mathrm{~Hz}$. (d) the velocity contour in the Z-direction during a quarter cycle at $100 \mathrm{~Hz}$. (e) the velocity contour in the X-direction during a quarter cycle at $5000 \mathrm{~Hz}$. (f-g) The deformation maps of piezoelectric transducer in the $+\mathrm{Z}$ direction with $100 \mathrm{~Hz}$ and $5000 \mathrm{~Hz}$ driving frequency.

the uniformity is broken below $5000 \mathrm{~Hz}$, and uneven deformation occurs in the perimeter in Fig. $6(\mathrm{~g})$. The reason is the vibration at high frequency is not sinusoidal and the deformation occurred as shown in Fig. 5.

\section{EXPERIMENT AND ANALYSIS}

\subsection{Experimental Investigation}

The vibrational performance of the piezoelectric transducers incorporated into the micropump was tested under conditions of both free and forced vibration using a PSV400 Scanning Vibrometer in Fig. 7(a). The experimental setup for performing these tests included a signal generator, a power amplifier, and an oscillograph, among other equipment, and is shown in Fig. 7(a). The flow rate and pressure of the micropump were tested using weighing and water-column methods, respectively in Fig. 7(b) and Fig. 7(c). The schematic of the measurements is shown in Fig. 7(d) that includes driving module, vibrational measurement module and flow rate, pressure measurement module. As shown in Fig. 7(e), nine measurement points were specified on the center plane (the diameter is $1 \mathrm{~mm}$ from the center to external circle) of the piezoelectric transducer in order to determine the distribution of vibrational displacements. The dynamic vibrational displacements of the transducer with $1 \mathrm{~mm}$ diameter of the PZT are shown in Fig. 7(f) on the display screen with the PSV 400 scanning vi- brometer. Here, the three different transducers with diameters of 12,15 , and $20 \mathrm{~mm}$ were studied under both uncoupled and coupled with the micropump conditions.

\subsection{Vibrational Displacement Measurement of the PZT}

The vibrational displacements of the nine measurement points were tested under both free-vibration and forcedvibration conditions. The maximum vibrational displacements of nine points are collected for transducers with $12 \mathrm{~mm}, 15 \mathrm{~mm}$ and $20 \mathrm{~mm}$ external diameter at a driving voltage of $40 \mathrm{Vpp}$, $70 \mathrm{Vpp}$ and $100 \mathrm{Vpp}$ of a sine-wave driving signal, respectively. The curve shapes of the maximum vibrational displacement are similar for all points from 1-9 under sine-wave driving according to analysis. For convenience, the average maximum displacements of nine points were calculated under every driving condition.

In order to examine the effect of driving voltage, Fig. 8 shows the maximum displacement versus frequency the piezoelectric transducers with external diameters of 12,15 , and $20 \mathrm{~mm}$ driven by a sine-wave signal at $100 \mathrm{Vpp}$ and three driving voltages 40,70 and $100 \mathrm{Vpp}$ of a sine-wave signal with $12 \mathrm{~mm}$ diameter. It can be seen that the average maximum displacements in Fig. 8(a) and Fig. 8(c) first increases, and then decreases under free-vibration as the frequency increases. The maximum displacement attains its greatest values near $60 \mathrm{~Hz}$, 


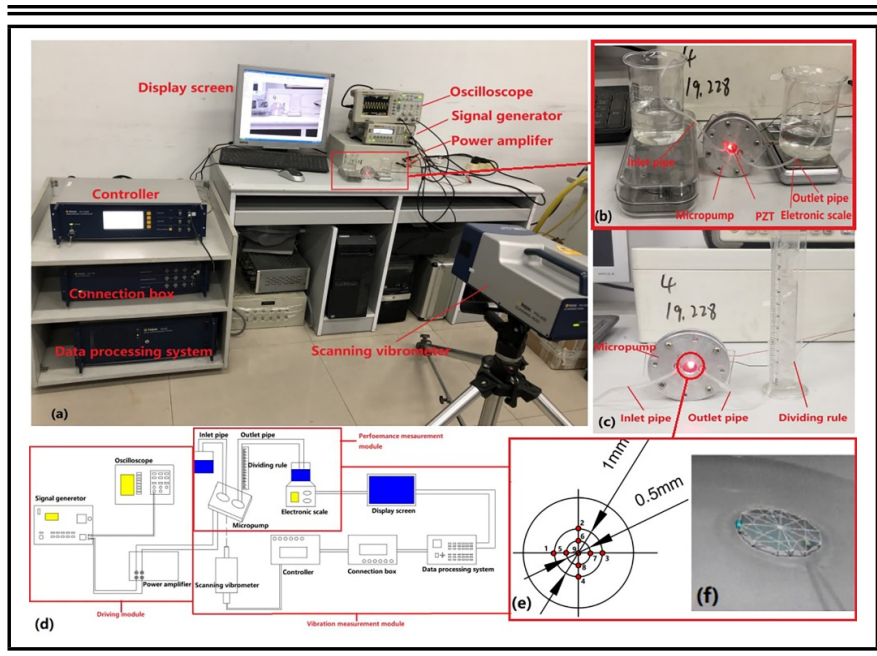

Figure 7. Layout of the experimental setup control system. (a) Photograph of vibration testing system for piezoelectric transducer. (b) The flow rate measurement of the micropump. (c) The pressure measurement of the micropump. (d) Schematic of the vibration displacement of the PZT, flow rate and pressure measurement of the micropump. (e) Distribution of the nine measurement points in the center plane during $1 \mathrm{~mm}$ of diameter from the center. (f) Dynamic vibrorecord displacements of the transducer during measuring.

which is the "resonant" frequency of the PZT at low driving frequency. At this frequency, the vibration displacement of PZT reaches the maximum value because the vibration is $1^{\text {st }}$ modal. Under $1^{\text {st }}$ modal vibration, the piezoelectric vibration in the $\mathrm{Z}$ direction is towards to the same direction, which is more suitable for the generation of vibration. At the same time the volume efficiency of the micropump should be the maximum value. Moreover, it can be seen from Fig. 8(c) that when the frequency is smaller (lower than $60 \mathrm{~Hz}$ ), the maximum displacement produced by PZT with larger diameter $(20 \mathrm{~mm})$ is larger than that with smaller diameter, which indicates that the resonant frequency of PZT decreases with the increase of diameter under low frequency driving. However, the average maximum displacements decrease as the frequency increases in Fig. 8(b) and Fig. 8(d). The maximum displacement is proportional to the voltage and inversely proportional to the diameter at the same frequency. The higher the voltage is, the greater the displacement is. The larger the diameter is, the greater the displacement is. Comparing with the simulation and experiment results under $40 \mathrm{Vpp}$ of driving voltage in Fig. 5 and Fig. 8(a), Fig. 8(b), the maximum displacement from experiment results emerged near $60 \mathrm{~Hz}$ or below $150 \mathrm{~Hz}$ and the value is smaller than the simulation one because the PZT vibration is obstructed after sealing with the lower PDMS and jig. Moreover, the maximum displacement is no longer increased along with the increase in the frequency (from $150 \mathrm{~Hz}$ to $800 \mathrm{~Hz}$ (even $5000 \mathrm{~Hz}$ )). That is because the displacement in the experiment is an average value (nine points in the diameter of $1 \mathrm{~mm}$ circle as shown in Fig. 7(e)) due to the limitation of experiment setup. However, the experiment results further prove the high frequency is not suitable as the working frequencies of the micropump. So lower frequencies (below $1000 \mathrm{~Hz}$ ) are adopted in the later experiments.

Comparing the free-vibration with the forced-vibration in Fig. 8(a), (c) and Fig. 8(b), (d), the behavior of the maximum displacements are different under the $100 \mathrm{Vpp}$ driving voltages with free-vibration and forced-vibration situations. The vibrational displacement under free vibration is higher than that under forced vibration over the entire frequency range. Because of the existence of the clamping boundary condition with

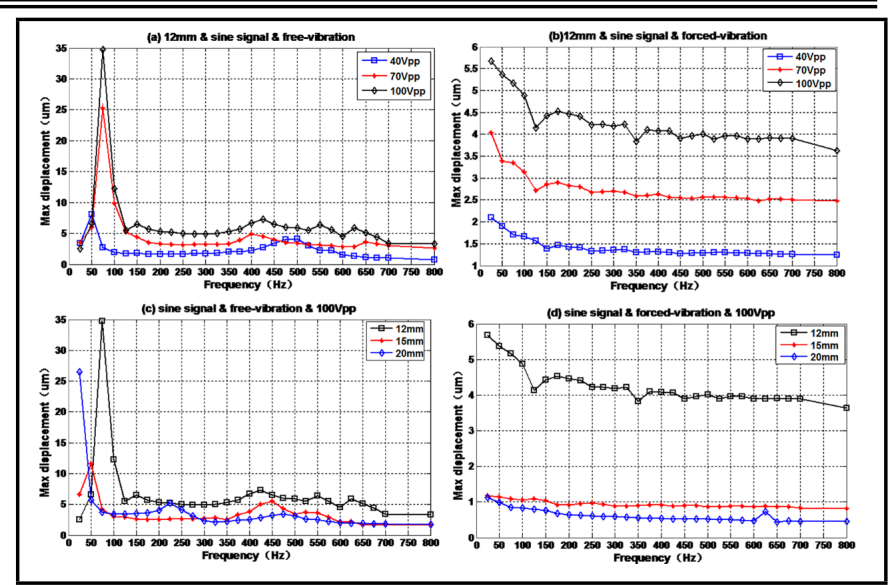

Figure 8. Maximum average displacements of the nine measurement points on the piezoelectric transducer versus frequency for different driving voltages and piezoelectric transducer diameters. (a) free-vibration with $40,70,100 \mathrm{Vpp}$ driving voltages. (b) forced-vibration with 40,70,100 Vpp driving voltages. (c) free-vibration with 12,15 and $20 \mathrm{~mm}$ piezoelectric transducer diameters. (d) forced-vibration with 12,15 and $20 \mathrm{~mm}$ piezoelectric transducer diameters.

the forced-vibration situation, the resonance frequency disappeared below a $100 \mathrm{~Hz}$ driving frequency. That is because the forces F1 and F2 from solid and fluid act as the dampers during the micropump operation according to Fig. 2. The displacements in the $\mathrm{X}$ and $\mathrm{Y}$ directions are close to zero under forced-vibration conditions once the piezoelectric transducer has been incorporated into the micropump. The nearer the measurement point is to the center of the transducer, the greater the vibrational displacement is. Thus, the displacements of the nine points under free-vibration are greater than those under forced-vibration in Fig. 8. Furthermore, it can be seen from Fig. 8 that the changing trend of the PZT displacement is basically the same before and after coupling, that is free-vibration and forced-vibration respectively. For example, the vibration performance with $100 \mathrm{Vpp}$ of voltage and $12 \mathrm{~mm}$ of diameter before coupling is the best, after coupling this situation is the same. This fully shows that the performance of PZT after coupling can be inferred from that before coupling, so as to simplify the detection steps, reduce the cost of testing, and achieve the optimization performance of the micropump for the future research.

\subsection{Flow Rate and Pressure Measurement of the Micropump}

Performance testing of the micropumps included measurements of the flow rate and pressure with different driving signals in Fig. 7(b). The flow rates (in practice, the maximum flow rates) of the micropumps with three diameters of piezoelectric transducer were measured by the same procedure. First, the inlet and outlet pipe were placed on the same plane, then the micropumps were actuated for $\Delta t=2$ min without any difference in height between the inlet and outlet reservoirs (zero pressure head). Finally, the mass of liquid in the outlet reservoir was determined using an analytical balance, and the flow rate was calculated as:

$$
\varnothing=\frac{W \times 10^{6}}{\rho \Delta t}(\mu L / \min )
$$

where $\varnothing$ is the flow rate, $W$ is the mass of liquid in the outlet reservoir (in $g$ ), and $\rho$ is the liquid density.

The pressures (in practice, the maximum pressure heads) of both micropumps were measured by placing the outlet pipe 
in a vertical position and measuring the liquid height, $\Delta \mathrm{Z}$, in Fig. 7(b), from which the pressure was calculated as:

$$
\Delta P=\Delta Z \rho g
$$

where $\Delta P$ is the pressure head at zero flow rate, and $g=$ $9.80 \mathrm{~m} / \mathrm{s}^{2}$ is the acceleration due to gravity.

The effects of driving voltage, transducer diameter and driving signal on flow rate and pressure are shown in Fig. 9. It can be seen that the behavior of the flow rate in Fig. 9(a), (c) and (e) and the pressure in Fig. 9(b), (d) and (f) are the same with the frequency. Both of them show a trend with first increasing, then decreasing, and then increasing and decreasing again as the frequency increases. There are two maximum flow rates and pressure values at $60 \mathrm{~Hz}$ and $600 \mathrm{~Hz}$ along the whole driving frequency. It further proves that the resonance frequency of the piezoelectric transducer before coupling will be changed after coupling. The optimal working condition of the micropump needs to be measured in detail and then conclusion can be deduced. The flow rate and pressure are proportional to the driving voltage. Both increase with increasing driving voltage in Fig. 9(a) and (b). The flow rate and pressure are higher for the transducer of $12 \mathrm{~mm}$ external diameter than for the 15and 20- mm transducers in Fig. 9(c) and (d); that means the flow rate and pressure are inversely proportional to the PZT diameters. This is in accordance with the values of the maximum displacement in Fig. 8(c) and (d). From Fig. 9(e) and (f), we can see that both flow rate and pressure are higher for the square-wave driving signal than for the sine-wave and trianglewave signals. Furthermore, the $12 \mathrm{~mm}$ diameter, square driving signal and $100 \mathrm{Vpp}$ driving voltage within the range of all measured diameters, driving signals and voltages show the best performance. These results are highly coincident with the numerical and vibrational results. At $100 \mathrm{Vpp}$ for the $12 \mathrm{~mm}$ diameter under sine-wave driving, the maximum flow rate and pressure are $150 \mu \mathrm{l} / \mathrm{min}$ and $346 \mathrm{~Pa}$ according to the experiment, respectively.

\section{CONCLUSIONS}

Three piezoelectric transducers with external diameters of 12,15 , and $20 \mathrm{~mm}$ were studied and designed for driving micropumps, and three kinds of micropumps with sandwich structures containing these transducers were fabricated using an advanced DRIE method on a silicon wafer. The optimal driving parameters of the piezoelectric transducer and micropump were determined by investigating their influence on the maximum vibrational displacement before and after coupling and under free- and forced-vibration conditions. Finally, experimental measurements of the three micropumps with different driving waves, driving voltages, and driving frequencies were carried out. The conclusions can be summarized as follows:

(1) The average displacements of the piezoelectric transducer after coupling with the pump body are smaller than those before coupling because of the counter force of the liquid. The vibration is steady and the deformation of the PZT is close to a sinusoidal trend under low frequencies (i.e., below $1000 \mathrm{~Hz}$ ).

(2) The trend of variation with frequency of the maximum displacement of the nine measurement points at the same driving voltage is the same for different driving waves for all three piezoelectric transducers. The maximum displacement under forced vibration is less than that under free vibration because of the fixed boundary conditions in the former case. The vibrational displacement near the center of the piezoelectric transducer is larger than elsewhere.

(3) Under both free- and forced-vibration conditions, the maximum displacement is inversely proportional to the diameters of the piezoelectric transducer and directly proportional to the driving voltage. The displacement reaches the maximum value under the square-wave driving signal, followed by the sine-wave signal, and then the triangle-wave signal. The optimal diameter of the PZT is $12 \mathrm{~mm}$. The performance of the micropump shows a good efficiency with square driving signal and $100 \mathrm{Vpp}$ driving voltage.

(4) The flow rate and pressure of the micropump show the same trends as the maximum displacement under the same driving conditions. Also, the trends of the experiment results coincide with the vibrational measurement results under the same driving conditions. There are two peak driving frequency values: $60 \mathrm{~Hz}$ and $600 \mathrm{~Hz}$. For the piezoelectric transducer of $12 \mathrm{~mm}$ diameter, under sine-wave driving at $100 \mathrm{Vpp}$, the maximum flow rate and pressure are $150 \mu \mathrm{l} / \mathrm{min}$ and $346 \mathrm{~Pa}$, respectively.

\section{REFERENCES}

1 M. Ashouri, MB. Shafii, Moosavi. A Diffuser miniature pump with an extra ferrofluidic valve. Microfluid Nanofluid, 19, 1235-1244, (2015). https://dx.doi.org/10.1007/s10404015-1642-9

2 A. Azarbadegan, CA. Cortes-Quiroz, I. Eames, Zangeneh M. Analysis of double chamber parallel valveless micropumps. Microfluid Nanofluid, 9, 171-180, (2010). https://dx.doi.org/10.1007/s10404-009-0519-1

3 VT. Dau, TX. Dinh, T. Katsuhiko, Susumu S. A cross-junction channel valveless-micropump with PZT actuation. Microsyst Technol, 15, 1039-1044, (2009). https://dx.doi.org/10.1007/s00542-009-0878-2

4 MW. Ashraf, S. Tayyaba, A. Nisar, et al. Design, Fabrication and Analysis of Silicon Hollow Microneedles for Transdermal Drug Delivery System for Treatment of Hemodynamic Dysfunctions. Cardiovasc Eng, 10, 91-108, (2010). https://dx.doi.org/10.1007/s10558-010-9100-5

5 Y. Li, W. Li, T. Guo, et al. Study on structure optimization of a piezoelectric cantilever with a proof mass for vibration-powered energy harvesting system. J. Vac. Sci. Technol. B, 27, 1288-1290, (2009). https://dx.doi.org/10.1116/1.3119677

${ }^{6} \mathrm{Z}$ Cheng, XD. Wu, J Cheng, et al. Microfluidic fluorescence-activated cell sorting ( $\mu$ FACS) chip with integrated piezoelectric actuators for low-cost mammalian cell enrichment. Microfluidics and Nanofluidics, 21(9), (2017). https://dx.doi.org/10.1007/s10404-017-1847-1

7 Z. Zhang, J. Kan, G. Cheng, et al. A piezoelectric micropump with an integrated sensor based on space-division multiplexing. Sensors Actuators A, 203, 29-36, (2013). https://dx.doi.org/10.1016/j.sna.2013.08.027 


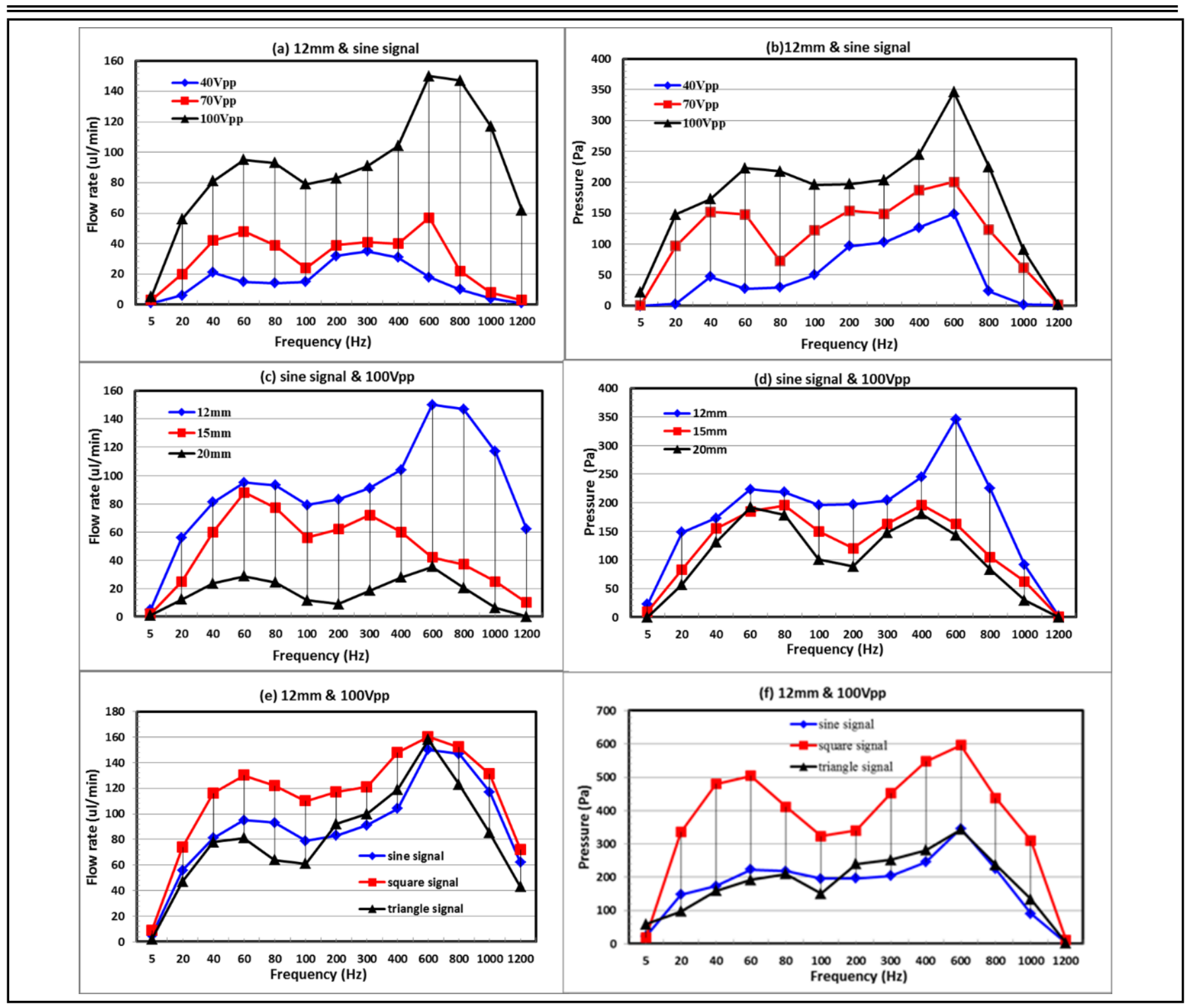

Figure 9. The flow rate and pressure of the micropump with different driving voltages, diameters of the PZT and driving signals. (a) Flow rate and (b) pressure versus frequency for the $12 \mathrm{~mm}$ diameter transducer with $40 \mathrm{Vpp}, 70 \mathrm{~V}$ pp and $100 \mathrm{Vpp}$ different driving voltages. (c) Flow rate and (d) pressure versus frequency for the 12, 15, and $20 \mathrm{~mm}$ diameter transducers at different driving voltages. (e) Flow rate and (f) pressure versus frequency for the 12 mm diameter transducer at different driving signals.

8 B. Pecar, D. Vrtacnik, D. Resnik, et al. Micropump operation at various driving signals: numerical simulation and experimental verification. Microsyst. Technol, 21, 1379-1384, (2015). https://dx.doi.org/10.1007/s00542014-2309-2

9 CN. Kim. Internal pressure characteristics and performance features of piezoelectric micropumps with the diffuser/nozzle and electromagnetic resistance. Comput. Fluids, 104, 30-39, (2014). https://dx.doi.org/10.1016/j.compfluid.2014.08.005

10 M. Sahin, FM Karadal, Y Y aman, et al. Smart structures and their applications on active vibration control: studies in the Department of Aerospace Engineering, METU. J. Electroceramics, 20, 167-174, (2008). https://dx.doi.org/10.1007/s10832-007-9130-6

11 Shuyu L. Analysis of the sandwich piezoelectric ultrasonic transducer in coupled vibration. J Acoust Soc Am, 117(2), 653-661, (2005). https://dx.doi.org/10.1121/1.1849960

12 X. Zhu, M. Zhao, Z. Gao, et al. Analysis of active vibration control for piezoelectric intelligent structures by ANASYS and MATLAB. Proceedings of ICCASM, 4, 184-188, (2010). https://dx.doi.org/10.1109/ICCASM.2010.5619058

13 SO.Catarino, L.R.Silva, P.M.Mendes et al. Piezoelectric actuators for acoustic mixing in microfluidic devices-Numerical prediction and experimental validation of heat and mass transport. Sensors and Actuators B: Chemical, 205(15), 206-214, (2014). https://dx.doi.org/10.1016/j.snb.2014.08.030

14 SC. Huang, CY. Tsai. Theoretical analysis of a new adjustable broadband PZT beam vibration energy harvester. Ultrasonics, 105, 304-314, (2016) . https://dx.doi.org/10.1016/j.ijmecsci.2015.11.027

15 S Waheed, Joan M. Cabot, Niall P. Macdonald, et al. 3D printed microfluidic devices: enablers and barriers. Lab on a chip, 16, 1993, (2016). https://dx.doi.org/10.1039/C6LC00284F

16 NT Nguyen, Xiaoyang Huang, Toh Kok Chuan. MEMS-Micropumps: A Review. Journal of Fluids Engineering, 124, 384-392, (2002). https://dx.doi.org/10.1115/1.1459075 
${ }^{17}$ E Yildirim, MAS. Arikan, H Kulah. A normally closed electrostatic parylene microvalve for micro total analysis systems. Sensors and Actuators A: Physical, 181, 81-86, (2012). https://dx.doi.org/10.1016/j.sna.2012.05.008

18 A. Ullmann, Y. Taitel. The piezoelectric valve-less pump: series and parallel connections. ASME J. Fluids Eng, 137, 021104-1-9, (2015). https://dx.doi.org/10.1115/1.4028534

19 A. Azarbadegan, C. Cortes-Quiroz, I. Eames, et al. Analysis of double-chamber parallel valve-less micropump. Microfluids Nanofluids, 9, 171-180, (2010). https://dx.doi.org/10.1016/10.1007/s10404-009-0519-1

${ }^{20}$ F. Amirouche, Y. Zhou, T. Johnson. Current micropump technologies and their biomedical applications. Microsyst. Technol. 15, 647-666, (2009). https://dx.doi.org/10.1007/s00542-009-0804-7

21 A. Nisar, NT. Afzulpurkar, BC. Mahaisavariya, at al. Mulifield analysis using multiple code coupling of a MEMS based micropump with biocompatible membrane for biomedical applications. BioMedical Engineering and Informatics, 1 ,531-535, (2008). https://dx.doi.org/10.1109/BMEI.2008.320

22 S Aggarwal, BE Paul, Amitava DasGupta, Dhiman Chatterjee. Experimental characterization of piezoelectrically actated micromachined silicon valveless micropump. Microfluid Nanofluid, 21, (2017). https://dx.doi.org/10.1007/s10404-016-1837-8

${ }^{23}$ CH Cheng, AS Yang, CJ Lin, WJ Huang. Characteristic studies of a novel piezoelectric impedance micropump. Microsystem Technology, 23(6), 1709-1717, (2017). https://dx.doi.org/10.1007/10.1007/s00542-015-2769-z

${ }^{24}$ XH. He, L. Xu, XT. Zhang and S. Yang. A bidirectional valveless piezoelectric micropump with three chambers applying synthetic jet. Journal of Mechanical Science and Technology, 30 (9), 4015-4022, (2016). https://dx.doi.org/10.1007/s12206-016-0814-1

25 B. Wang, X. Chu, E. Li, et al. Simulation and analysis of a piezoelectric micropump. Ultrasonics, 44, 643-646, (2006). https://dx.doi.org/10.1016/j.ultras.2006.05.018

26 J. Kang, GW. Auner. Simulation and verification of a piezoelectrically actuated diaphragm for check valve micropump design. Sensors Actuators A, 167, 512-516, (2011). https://dx.doi.org/10.1016/j.sna.2011.01.012

27 S. Singh, N. Kumar, D. George. Analytical modeling, simulations and experimental studies of a PZT actuated planar valveless PDMS micropump. Sensors Actuators A, 225, 81-94, (2015). https://dx.doi.org/10.1016/j.sna.2015.02.012

${ }^{28}$ Zhang ZH, Kan JW, Wang SY, Wang HY, Ma JJ, Jiang YH. Development of self-sensing piezoelectric pump with a bimorph transducer. Journal of intelligent material systems and structures, 27(5), 581-591, (2016). https://dx.doi.org/10.1177/1045389x15575082

29 Q. Guo, GZ. Cao, IY. Shen. Measurements of piezoelectric coefficient $\mathrm{d} 33$ of lead zirconate titanate thin films using a mini force hammer. ASME J. Vib. Acoust, 135(1), 0110031-9, (2013). https://dx.doi.org/10.1115/1.4006881
${ }^{30} \mathrm{R}$ Koven, $\mathrm{M}$ Mills, $\mathrm{R}$ Gale, et al. Low-Frequency and Broadband Vibration Energy Harvesting Using BaseMounted Piezoelectric Transducers. IEEE Trans Ultrason Ferroelectr Freq Control, 64(11), 1735-1743, (2017). https://dx.doi.org/10.1109/TUFFC.2017.2739745

31 S Khazaaleh, N Saeed, I Taha et al. Piezoelectric micromachined ultrasonic transducers and micropumps: from design to optomicrofluidic applications. Proceeding of SPIE, 10061 (7), 133-139, (2017). https://dx.doi.org/10.1117/12.2254733

32 CJ. Lee, HJ. Sheen, ZK. Tu, et al. A study of PZT valveless micropump with asymmetric obstacles. Microsyst. Technol, 15, 993-1000, (2009). https://dx.doi.org/10.1007/s00542009-0839-9

${ }^{33}$ SY. Lin. An improved cymbal transducer with combined piezoelectric ceramic ring and metal ring. Sensors and Actuators A, 163, 266-276, (2010). https://dx.doi.org/10.1016/j.sna.2010.06.022

${ }^{34}$ Y. Guan, C. Liu. Structural parameter analysis and experimental study of micropumps with saw-tooth microchannel. Sensors Actuators A, 234 (2015) 195-205. https://dx.doi.org/10.1016/j.sna.2015.09.003

35 J. Ying, C. Cao, Z. Jiao. Loss coefficient and rectification efficiency based on valveless micropump. $J$. Zhejiang Univ. (Eng. Sci.), 47, 249-255, (2013). https://dx.doi.org/10.3785/j.issn.1008-973X.2013.02.009

${ }^{36}$ Q. Yao, D Xu, LS. Pan, at al. CFD simulations of flows in valveless micropumps. Engineering applications of computational fluid mechanics, 1(3), 181-187, (2007). https://dx.doi.org/10.1080/19942060.2007.11015191

37 J. Xu, T. Guo, H. Sun, et al. Analysis of a piezoelectric micropump by using ANSYS. Proceedings of Symposium on Piezoelectricity, Acoustic Waves, and Device Applications, 370-373, 2008. https://dx.doi.org/10.1109/SPAWDA.2008.4775812

38 A. Bybi, S. Grondel, J. Assaad, et al. Reducing crosstalk in array structures by controlling the excitation voltage of individual elements: a feasibility study. Ultrasonics, 53, 1135-1140, (2013). https://dx.doi.org/10.1016/j.ultras.2013.02.009

39 NK. Karadimitriou, V. Joekar-Niasar, S. M. Hassanizadeh, et al. A novel deep reactive ion etched (DRIE) glass micromodel for two-phase flow experiments. Lab Chip, 12, 3413-3418, (2012). https://dx.doi.org/10.1039/c2lc40530j

${ }^{40}$ MX Guo, XJ Chou, JL Mu, et al. Fabrication of microtrench structures with high aspect ratio based on DRIE process for MEMS device applications. Microsyst Technol, 19, 1097-1103, (2013). https://dx.doi.org/10.1007/s00542012-1720-9

41 J. Sirohi and I. Chopra. Fundamental understanding of piezoelectric strain sensors. J. Intell. Mater. Syst. Smart Struct, 11, 246-257, (2000). https://dx.doi.org/10.1106/8BFB-GC8P-XQ47-YCQ0

${ }^{42}$ M. Deluca, T. Sakashita, C. Galassi, G. Pezzotti. Investigation of local orientation and stress analysis of pzt-based materials using micro-probe polarized raman spectroscopy. Journal of the European Ceramic Society, 26(12), 2337-2344, (2006). https://dx.doi.org/10.1016/j.jeurceramsoc.2005.04.016 